\section{AB0541 LUPUS MANIFESTATIONS IN A PATIENT WITH SPONDYLOARTHRITIS TREATED WITH TUMOUR NECROSIS FACTOR-ALPHA ANTAGONIST: SYSTEMIC LUPUS ERYTHEMATOSUS (SLE) OR DRUG-INDUCED LUPUS ERYTHEMATOSUS (DILE)?}

Mariam Hamid Mustapha ${ }^{1,1}$, Syang Pyng Gan ${ }^{1}$, Hazlyna Baharuddin ${ }^{2}$, Norliza Zainudin ${ }^{1}$, Shereen Suyin Ch'ng ${ }^{1}{ }^{1}$ Hospital Selayang, Rheumatology Unit, Department of Medicine, Selangor, Malaysia; ${ }^{2}$ Universiti Teknologi MARA (UiTM), Faculty of Medicine, Selangor, Malaysia

Background: Systemic lupus erythematosus (SLE) is a multisystem autoimmune inflammatory disease. Drug-induced lupus erythematosus (DILE) is defined as the development of lupus-like symptoms that is temporarily related to continuous drug exposure which resolves with cessation of the offending drug. It is usually accompanied by serologic findings of a positive antinuclear antibody (ANA) and anti-histone antibodies. Tumour necrosis factor- $\alpha$ (TNF $\alpha$ ) antagonist-induced lupus-like syndrome (TAILS) is a well-known side effect of this class of substances. A retrospective study reported twenty two cases whose lupus manifestations abated within a few weeks (median eight weeks) in all patients except one with longer-lasting evolution of six months.

Objectives: We reported a case which posed a diagnostic challenge to us, a patient with spondyloarthritis $(\mathrm{SpA})$ who presented with lupus-like features during treatment with Golimumab.

Methods: Case report

Results: A 36-year-old lady was diagnosed as spondyloarthritis when she presented with peripheral arthritis, plantar fasciitis, right eye anterior uveitis and bilateral chronic sacroilitis confirmed via sacroiliac magnetic resonance imaging. Investigations revealed raised erythrocyte sedimentation rate (ESR) and negative HLA-B27. She developed allergic reaction to sulfasalazine and non-steroidal anti-inflammatory drugs (NSAIDS), thus intravenous Golimumab was initiated. Although she responded to this therapy and ESR had reduced during assessment at 24 weeks, Golimumab was stopped due to funding issue. It was restarted after six months but had to be discontinued again after four months because she developed recurrent fever and malar rash. During this presentation, investigations revealed leukopenia of $2.46 \times 10^{9}$, ESR of $93 \mathrm{~mm} / \mathrm{hr}$, low complements and positive direct coombs' test. ANA, anti-double stranded DNA, anti-histone antibody and anti-smith antibody were all negative. Infective screening was negative. TAILS secondary to Golimumab was diagnosed. Apart from discontinuation of Golimumab, $0.5 \mathrm{mg} / \mathrm{kg} / \mathrm{day}$ of prednisolone was started which resulted in improvement of the malar rash and leukopenia. However, four months after the last dose of Golimumab, recurrent fever and leukopenia recurred when the prednisolone dose was reduced to $5 \mathrm{mg}$ on alternate-days. At this time, we considered diagnosis of SLE rather than TAILS because of persistent SLE manifestation despite cessation of Golimumab and improvement with oral corticosteroid. Furthermore, although not pathognomonic, the anti-histone antibody was negative.

Conclusion: This case posed a diagnostic challenge as both SLE and DILE can present similarly. The approach to managing this patient with coexisting SpA and SLE is also a challenge since there is a dilemma as to what can be offered after failure of NSAIDs and conventional disease-modifying antirheumatic drugs since anti-TNF $\alpha$ is contraindicated in SLE.

\section{REFERENCES}

[1] Vasoo S. Drug-induced lupus. Lupus, 2006; 15: 757-761

[2] Brussano AM, Aberer W, Massone C. Subacute lupus erythematosus during treatment with golimumab for seronegative rheumatoid arthritis. Lupus, 2014; 23: 201-3.

[3] De Bandt M, Sibilia J, Le Loet X, et al. Systemic lupus erythematosus induced by anti-tumour necrosis factor alpha therapy: A French National survey. Arthritis Res Ther, 2005; 7: R545-51

[4] Oscar M. Pérez-Fernández, et al. Spondyloarthropathies in Autoimmune Diseases and Vice Versa. Autoimmune Diseases, 2011: 2012

Disclosure of Interests: None declared DOI: 10.1136/annrheumdis-2019-eular.4542

\section{$\mathrm{AB} 0542$ \\ PREGNANCY OUTCOMES IN ANTIPHOSPHOLIPID SYNDROME: 8 YEAR-EXPERIENCE FROM A MULTIDISCIPLINARY UNIT}

Agna Neto ${ }^{1,2}$, Patrícia Martins ${ }^{3,4}$, Susana Capela $a^{3,4}$, Claudia Araujo ${ }^{5}$, Mónica Centeno ${ }^{5}$, Manuela Costa ${ }^{1}$, Luísa Pinto ${ }^{5} .{ }^{1}$ Reumatologia, Hospital de Egas Moniz, CHLO, Lisboa, Portugal; ${ }^{2}$ Reumatologia, Hospital Central do Funchal, Madeira, Portugal; ${ }^{3}$ Reumatologia e Doenças Ósseas Metabólicas, Centro Académico de Medicina de Lisboa, Lisboa, Portugal; ${ }^{4}$ Instituto de Medicina Molecular, FMUL, Centro Académico de Medicina de Lisboa, Lisboa, Portugal; ${ }^{5}$ Departamento de Ginecologia, Obstetrícia e Medicina da Reprodução, Hospital de Santa Maria, CHLN, Lisboa, Portugal

Background: Women with antiphospholipid syndrome (APS) are at increased risk of recurrent miscarriage, fetal death, placental insufficiency, preeclampsia and fetal growth restriction. Although treatment improves fetal-maternal outcomes, there are still some unsuccessful pregnancies. A multidisciplinary approach with strict monitoring is essential in order to attain obstetrical success.

Objectives: To assess pregnancy outcomes in portuguese women with APS who were surveilled at a multidisciplinary unit.

Methods: Pregnant women fulfilling the Sydney classification criteria for definite APS, who attended our specialized Rheumatology and Obstetrics outpatient clinic between 2010 and 2018, were included in this retrospective observational study. Cases of suspected APS not meeting the classification criteria were excluded. All pregnancies were followed by a multidisciplinary team (rheumatologists, obstetricians and nurses). Data was collected from medical records. Adverse Pregnancy Outcomes (APO) were defined as: spontaneous abortion $(<10 \mathrm{w})$, fetal death $(\geq 10 \mathrm{w})$, neonatal death, fetal growth restriction (FGR) and delivery prior to 36 weeks of gestation with or without preeclampsia (PE).

Results: A total of 35 pregnancies were identified in 25 women with APS. Twelve (48\%) patients had thrombotic APS, $9(36 \%)$ had obstetric APS and $4(16 \%)$ had mixed APS. Primary APS was seen in $56 \%$ of patients, while systemic lupus erythematosus was found in $44 \%$.

The average maternal age at conception was $32.8 \pm 5.2$ years. Mean duration of disease prior to pregnancy was $6.4 \pm 5.5$ years. In regard to antiphospholipid antibody (APL) profile, $28.6 \%, 25.7 \%$ and $28.6 \%$ of patients were triple, double and single positive, respectively. Although they had fulfilled laboratorial criteria in the past, $17 \%$ of patients were negative for all APL. All patients were instructed to receive prophylactic or therapeutic low-molecular-weight heparin combined with low dose aspirin for the duration of pregnancy.

Regarding fetal outcomes, there were $2(5.7 \%)$ cases of first-trimester miscarriage, $1(2.9 \%)$ medical abortion due to exposure to teratogenic drugs at the time of conception and $4(11.4 \%)$ fetal deaths. Among the cases of fetal death, one concerned a patient who suspended heparin on her own initiative and another one who became pregnant under warfarin and whose fetus had trisomy 18. The other cases occurred at 11 and 18 weeks of gestation, under regular therapy. There were no cases of neonatal death or other fetal malformations. The rate of live births was $80 \%$, with a mean gestational age of $37.3 \pm 1.5$ weeks and mean birth weight of $2796.4 \pm$ $462 \mathrm{~g}$. Most women delivered by cesarean section $(54.3 \%$ of cases). There were $6(17.1 \%)$ cases of preterm birth, three $(8.6 \%)$ corresponding to fetus with FGR. Concerning maternal outcomes, there was one single case $(2.9 \%)$ of PE. There were no cases of eclampsia or HELLP syndrome.

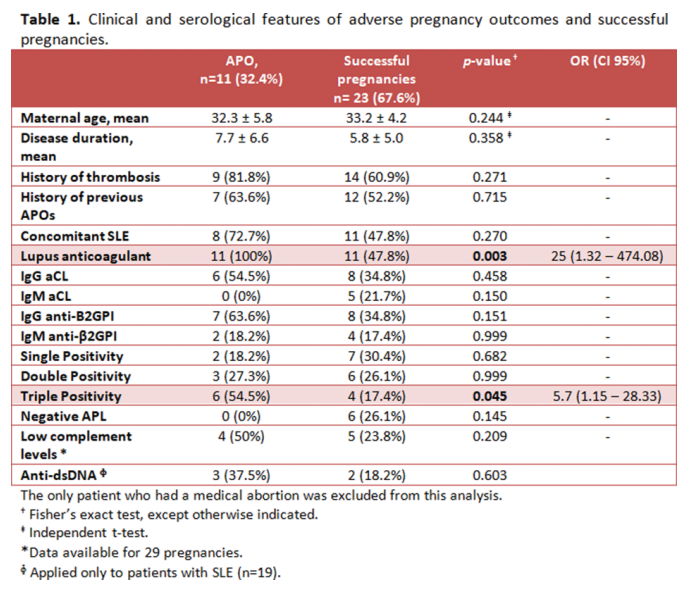

Lupus anticoagulant $(p=0.003, \mathrm{OR} 25, \mathrm{Cl} 95 \% 1.32-474.0)$ and triple APL $(p=0.045$, OR $5.7, \mathrm{Cl} 95 \% 1.15-28.33$ ) positivity were associated with adverse pregnancy outcomes. In this cohort, no association was 
found between poor obstetric outcomes and history of thrombosis, presence of SLE or low complement levels (table 1).

Conclusion: In our study, most pregnancies were uneventful. Despite the small sample size, we reinforce the importance of a multidisciplinary evaluation and surveillance before, during and after pregnancy in women with APS in order to implement early treatment and to optimize fetal-maternal outcomes.

Disclosure of Interests: None declared

DOI: 10.1136/annrheumdis-2019-eular.1079

\section{AB0543 1 FEATURES ASSOCIATED WITH RENAL DAMAGE IN PATIENTS WITH SYSTEMIC LUPUS ERYTHEMATOSUS DECEASED OVER A 10-YEAR PERIOD}

Ivan Padjen ${ }^{1}$, Marijan Erceg ${ }^{2}$, Mislav Cerovec ${ }^{1}$, Miroslav Mayer ${ }^{1}$,

Ranko Stevanovic ${ }^{2}$, Branimir Anic ${ }^{1} .{ }^{1}$ University Hospital Centre Zagreb, University of Zagreb School of Medicine, Division of Clinical Immunology and Rheumatology, Department of Internal Medicine, Zagreb, Croatia; ${ }^{2}$ Croatian Institute of Public Health, Zagreb, Croatia

Background: Renal damage (RD) is one of the most important contributors to morbidity and mortality in patients with systemic lupus erythematosus (SLE).

Objectives: We aimed to assess features associated with RD in a group of 90 deceased SLE patients routinely followed-up at our institution, which serves as a national referral center for SLE.

Methods: We retrospectively analyzed 90 SLE patients (68 females) deceased from 2002 to 2011. All patients were $\geq 18$ years of age at death, fulfilling $\geq 4 / 11$ classification criteria of the American College of Rheumatology (ACR). We identified patients with RD, as defined by the Systemic Lupus International Collaborating Clinics (SLICC)/ACR index. An extensive set of variables was compared between patients with and without RD (RD and RD-N, respectively): demographics, ACR criteria at diagnosis and cumulatively at death, total damage and its components one year following diagnosis and non-renal damage and its components cumulatively at death, as well as components of the metabolic syndrome, smoking, sicca and Hughes syndrome. Frequencies were compared using the chi-square and Fisher's exact test, and continuous variables using the t-test and Mann-Whitney $U$ test. Variables associated with RD were analyzed using multivariate logistic regression.

Results: We identified 25/90 patients who accrued RD over the course of their disease. In the univariate analysis, we found no difference between $\mathrm{RD}$ and RD-N patients in any of the following parameters: demographics, total count of ACR criteria at diagnosis and death, as well as damage at one year after diagnosis and cumulative non-renal damage at death. Compared to RD-N patients, RD patients had a higher proportion of malar rash at diagnosis $(11 / 25$ vs. $13 / 65, p=0.021)$ and a higher cumulative proportion of renal disorder (19/25 vs. $30 / 65, p=0.011$ ), including proteinuria and urinary casts (17/25 vs. $23 / 65, p=0.005$, for both). RD patients also had a higher proportion of myocardial infarction as an item of cumulative damage $(7 / 25$ vs. $6 / 65, p=0.023)$ and were more frequently obese (11/25 vs. 15/65, $\mathrm{p}=0.049)$. Conversely, hematological disorder and leukopenia at diagnosis were less frequent in RD compared to RD-N patients (4/25 vs. $30 / 65, \quad \mathrm{p}=0.008$ and $1 / 25$ vs. $21 / 65$, respectively). In the final multivariate model (adjusted for gender, age at diagnosis and disease duration), malar rash at diagnosis and the cumulative presence of renal disorder (classification criteria of the ACR) were positively associated with RD. Conversely, leukopenia at diagnosis was inversely associated with RD (Figure 1).

\begin{tabular}{lccc} 
Variable & OR & $95 \% \mathrm{Cl}$ & $\mathrm{p}$ \\
\hline Malar rash (dg)(yes/no) & $\mathbf{2 7 . 4 1 6}$ & $\mathbf{3 . 5 8 6 - 2 0 9 . 6 2 0}$ & $\mathbf{0 . 0 0 1}$ \\
Leukopenia (dg)(yes/no) & $\mathbf{0 . 0 1 6}$ & $\mathbf{0 . 0 0 1 - 0 . 2 7 3}$ & $\mathbf{0 . 0 0 4}$ \\
Renal disorder (cum)(yes/no) & $\mathbf{7 . 1 4 1}$ & $\mathbf{1 . 0 9 1 - 4 6 . 7 6 0}$ & $\mathbf{0 . 0 4 0}$ \\
Myocardial infarction (cum)(yes/no) & 6.117 & $0.319-117.197$ & 0.229 \\
Obesity (cum)(yes/no) & 1.917 & $0.425-8.638$ & 0.397 \\
Male gender (yes/no) & 1.913 & $0.335-10.916$ & 0.465 \\
Age (dg)(years, cont) & 1.018 & $0.969-1.070$ & 0.481 \\
Disease duration (cum)(years, cont) & 0.940 & $0.826-1.070$ & 0.352 \\
\hline
\end{tabular}

$\mathrm{dg}$ - at diagnosis, cum - cumulatively, yes/no - binary variable, cont - continuous variable

Figure 1. Features associated with renal damage (multivariate logistic regression model)
Conclusion: More than a quarter of deceased patients accrued RD. While malar rash at diagnosis may be associated with a higher likelihood of developing RD, early leukopenia may be associated with its lower likelihood in deceased patients.

\section{REFERENCES}

[1] Davidson A. Nat Rev Rheumatol. 2016;12:143-53.

Disclosure of Interests: Ivan Padjen: None declared, Marijan Erceg: None declared, Mislav Cerovec: None declared, Miroslav Mayer Speakers bureau: Novartis, Sandoz, Abbvie, Pfizer, Alvogen, Roche, MSD, Octapharma, Ranko Stevanovic: None declared, Branimir Anic Speakers bureau: Novartis, Sandoz, Abbvie, Pfizer, Alvogen, Roche, MSD, Octapharma

DOI: 10.1136/annrheumdis-2019-eular.2885

\section{AB0544 ECHOCARDIOGRAPHIC CHANGES IN PATIENTS WITH SYSTEMIC LUPUS ERYTHEMATOSUS BEFORE TO INITIATION OF IMMUNOSUPPRESSIVE THERAPY}

Tatiana Panafidina ${ }^{1}$, Tatiana Popkova ${ }^{2}$, Alexander Volkov ${ }^{3} .{ }^{1}$ V.A.Nasonova Research Institute of Rheumatology, Systemic rheumatic diseases, Moscow, Russian Federation; ${ }^{1}$ V.A.Nasonova Research Institute of Rheumatology, Systemic rheumatic diseases, Moscow, Russian Federation; ${ }^{3}$ V.A.Nasonova Research Institute of Rheumatology, Diagnostic department, Moscow, Russian Federation

Background: Cardiovascular diseases are becoming the leading cause of death among SLE patients due to increasing life-spans. Transthoracic echocardiography (TTE) is a routine and widely available modality in everyday clinical practice useful to identify specific pathological cardiac changes and predictors of heart failure (HF).

Objectives: Obtaining of specific TTE findings in SLE patients prior to initiation of pathogenic immunosuppressive therapy was the objective in this study. Methods: Thirty four pts (91\% females, aged 30[26-34]years (median [interquartile range 25\%-75\%]) with "non-treated" SLE (ACR 1997 and SLICC 2012 criteria) were included. None of pts was treated either with prednisone or cytotoxic drugs at the moment of inclusion.

Results: Median SLE duration was 18[6-60]months, SLEDAI-2K - 13[8-19], SLICC/DI - 0[0-0]scores. Leading SLE clinical manifestations included: hematological changes $(74 \%)$, kidney involvement $(59 \%)$, joints $(50 \%)$ and skin involvement $(50 \%)$. Immunological abnormalities were detected in all patients and were as follows: ANA positivity - in 100\%, anti-dsDNA antibodies - in $76 \%$ of SLE patients. Concurrent antiphospholipid syndrome was found in $2(6 \%)$ patients. Valve insufficiency with varying degree of regurgitation was the commonest pathology found in "non-treated" SLE patients based on TTE data: mitral valve insufficiency - in 31(91\%), tricuspid valve - in $31(91 \%)$, pulmonary valve insufficiency - in $21(62 \%)$, aortic valve insufficiency - in $4(12 \%)$ patients. Endocarditis was a rare pathology found in $5(15 \%)$ patients, while mitral and tricuspid valves prolapse was seen more often - in 16(47\%), while not a single case of valve stenosis was found. Pericardial pathology was detected in $16(47 \%)$ patients: exudative - in $9(26 \%)$, and adhesive (thickening, hardening and separation of leaflets) - in $7(21 \%)$

There were no cases of CAD or $\mathrm{Ml}$, although there were $2(6 \%)$ documented cases of cerebral stroke in past history, and $1(3 \%)$ case of confirmed CHF. Most common TRF were dyslipidemia and hypertension - in $15(44 \%)$ and $11(32 \%)$ SLE patients respectively.

Median LVEF was 64[59-67]\%, LV end-systolic dimension- 30[27-32]mm, LV end-diastolic dimension - 48[45-51]mm, pulmonary artery systolic pressure - 24[22-32] $\mathrm{mm} \mathrm{Hg}$. LV diastolic disfunction (LVDD) was found in 10 (29\%), systolic dysfunction (LVSD) - in 4(12\%), LV myocardial hypertrophy $(\mathrm{LVH})$ - in $5(15 \%)$; left atrium dilatation (LAD) was found in $4(12 \%)$, and increased dimensions of right atrium was detected in 3(9\%) SLE patients.

Conclusion: Most common cardiac abnormalities in "non-treated" SLE patients with high activity (SLEDAI-2K 13 scores) were valve dysfunction (insufficiency with regurgitation), mitral and tricuspid valve prolapse and pericarditis. Of importance is the presence of early subclinical features of HF almost in $1 / 3$ of naïve to treatment SLE patients: LVDD (29\%), LVH $(15 \%)$, and LAD (12\%). SLE patients should be thoroughly monitored both for adequate control of SLE activity, and cardiac pathology with correction of TRF, regular assessments by a cardiologist, TTE, and early administration of cardio-protection therapy in view of increased HF risk in SLE patients, predetermining unfavorable prognosis.

Disclosure of Interests: None declared

DOI: 10.1136/annrheumdis-2019-eular.7094 